\title{
Sleep in Offspring of Parents With Mood Disorders
}

\author{
Delainey L. Wescott ${ }^{1,2}$, Jessica Morash-Conway ${ }^{3}$, Alyson Zwicker ${ }^{3,4}$, Jill Cumby $^{3}$, \\ Rudolf Uher ${ }^{1,2,3}$ and Benjamin Rusak ${ }^{1,2,3 *}$ \\ ${ }^{1}$ Department of Psychology and Neuroscience, Dalhousie University, Halifax, NS, Canada, ${ }^{2}$ Department of Psychiatry, \\ Dalhousie University, Halifax, NS, Canada, ${ }^{3}$ Nova Scotia Health Authority, Halifax, NS, Canada, ${ }^{4}$ Department of Pathology, \\ Dalhousie University, Halifax, NS, Canada
}

Background: Sleep problems in childhood are an early predictor of mood disorders among individuals at high familial risk. However, the majority of the research has focused on sleep disturbances in already diagnosed individuals and has largely neglected investigating potential differences between weeknight and weekend sleep in high-risk offspring. This study examined sleep parameters in offspring of parents with major depressive disorder or bipolar disorder during both weeknights and weekends.

\section{OPEN ACCESS}

Edited by:

Baoman Li,

China Medical University,

China

Reviewed by:

Timo Partonen,

National Institute for Health and Welfare,

Finland

Axel Steiger,

Max-Planck-Institut für Psychiatrie,

Germany

${ }^{*}$ Correspondence:

Benjamin Rusak

Benjamin.Rusak@dal.ca

Specialty section:

This article was submitted to

Sleep and Chronobiology,

a section of the journal

Frontiers in Psychiatry

Received: 06 February 2019

Accepted: 26 March 2019

Published: 08 April 2019

Citation:

Wescott DL, Morash-Conway J, Zwicker A, Cumby J, Uher R and Rusak B (2019) Sleep in Offspring of Parents With Mood Disorders.

Front. Psychiatry 10:225.

doi: 10.3389/fpsyt.2019.00225
Methods: We used actigraphy, sleep diaries, and questionnaires to measure several sleep characteristics in 73 offspring aged 4-19 years: 23 offspring of a parent with major depressive disorder, 22 offspring of a parent with bipolar disorder, and 28 control offspring.

Results: Offspring of parents with major depressive disorder slept, on average, 26 min more than control offspring on weeknights (95\% confidence interval, 3 to 49 min, $p=$ 0.027). Offspring of parents with bipolar disorder slept, on average, 27 min more on weekends than on weeknights compared to controls, resulting in a significant family history $\times$ weekend interaction (95\% confidence interval, 7 to $47 \mathrm{~min}, p=0.008$ ).

Conclusions: Sleep patterns in children and adolescents were related to the psychiatric diagnosis of their parent(s). Future follow-up of these results may clarify the relations between early sleep differences and the risk of developing mood disorders in individuals at high familial risk.

Keywords: sleep, severe mental illness, major depressive disorder, bipolar disorder, actigraphy, high-risk offspring, cohort study

\section{INTRODUCTION}

Sleep disturbances are core symptoms of mood disorders including major depressive disorder and bipolar disorder (1). Additionally, sleep problems have been associated with more severe symptoms, greater functional impairment, and increased risk for relapse among individuals with mood disorders (2). Over $40 \%$ of children and youth experience sleep disturbances during development (3). Early sleep disturbances have been shown to predict depressive symptoms (4, $5)$, manic episodes $(6,7)$, and psychotic-like experiences (8). However, there have been some inconsistencies in the reported relationship between sleep and psychopathology (9). The persistence of sleep problems may represent a key factor in the true relationship between sleep disturbance in childhood and later mental illness. Many sleep problems resolve during development, but those that persist predict symptoms of depression, anxiety, and externalizing behaviors into adulthood (10). 
These findings highlight the importance of treating sleep disturbances as a contributing factor to, and not just a symptom of, mood disorders, and emphasize the need for longitudinal investigations of these relationships.

Many previous studies examining the relationship between sleep disturbances and mood disorders focused on individuals who had already been diagnosed (11). There has been less research examining sleep disturbances among children and youth at high familial risk of mood disorders. This gap in the literature is surprising given that offspring of parents with a mood disorder have a 1 in 3 chance of developing a mental illness themselves (12).

Mood disorders typically have an onset in the second or third decade of life; however, they are often preceded by earlier manifestations, which have been referred to as antecedents (13), defined as conditions that precede the onset of mood disorders by at least several years (14). Longitudinal high-risk studies suggest that sleep disturbances likely represent an antecedent to mood disorders (14-16). However, there is limited information on early sleep disturbances among offspring of parents with mood disorders. Offspring of mothers with postpartum depression are more likely than control offspring to have sleep problems (17) and to develop depression themselves (18). Consistent with these findings, there is evidence that sleep disturbances are prevalent among offspring of a parent with bipolar disorder $(7,19,20)$.

Previous research on sleep in high-risk offspring has relied largely on self-reported measures of sleep $(7,14)$ despite the known benefits of combining self-report and objective measures (21). Actigraphy is an objective, indirect measure of sleep that uses accelerometer-based devices to estimate sleep parameters (22) and provides an ecologically valid representation of typical sleep patterns in a participant's natural environment (23). Studies that have used objective sleep measures have typically not reported sleep data from weeknight and weekends separately, despite recommendations to do so $(24,25)$. Discrepancies in sleep patterns between weeknights and weekends are prevalent during adolescence. These discrepancies are linked to emotional disturbances and have been shown to be independently associated with self-harm among adolescents (26). Additionally, sleep duration on free days (e.g., weekends or vacation) has been shown to be more heritable than sleep duration on sleepconstrained days (e.g., weeknights preceding school days) (27), and variability in weeknight and weekend sleep is a common sleep pattern observed in individuals with bipolar disorder (14). This reinforces the importance of evaluating these sleep parameters on weeknights and weekends separately among offspring at high familial risk of mood disorders.

The current study used actigraphy, sleep diaries, and selfreport sleep questionnaires to examine sleep among offspring of parents with mood disorders during both weeknights and weekends. Sleep period length, total sleep time (TST), sleep onset latency (SOL), and wake after sleep onset (WASO) were compared between offspring at high familial risk of mood disorders and offspring of control parents. We hypothesized that children and youth at familial risk of developing a mood disorder would have more disturbed sleep than offspring of controls, as indicated by longer SOLs, increased WASO, and larger weeknight-weekend discrepancies in TST.

\section{MATERIALS AND METHODS}

\section{Sample Description}

We measured sleep in children and youth aged 4-19 years who participate in the Families Overcoming Risks and Building Opportunities for Well-being (FORBOW) cohort, which includes offspring of parents with severe mental illness and compares them to controls with no known familial risk (28).

Offspring of parents with mood disorders were recruited from February 2015 to June 2018 through their parents' contact with mental health services in Nova Scotia, Canada. Offspring of control parents matched for age and socioeconomic status were recruited through local school boards. In this study, we included offspring with a parent diagnosed with major depressive disorder or bipolar disorder, as well as offspring of control parents. Participants and at least one parent were required to have the capacity to read, write, and speak English. We excluded youth with severe intellectual disability or autism spectrum disorders. No exclusions were made based on sex, ethnicity, living arrangements, medication, or other psychopathology.

\section{Parent Assessment}

Diagnoses of mental disorders according to the Diagnostic and Statistical Manual of Mental Disorders, Fourth Edition (DSM-IV) and DSM-5 were established using the Schedule for Affective Disorders and Schizophrenia (SADS for DSM-IV) and the Structured Clinical Interview for DSM-5 Disorders (SCID-5) (29). Diagnoses were confirmed in consensus meetings with a psychiatrist blind to offspring psychopathology. Offspring were placed in family history groups based on their biological parent's diagnosis. In cases where offspring had two parents with different diagnoses, they were placed in the family history group based on the DSM-5 diagnostic hierarchy. Thus, offspring with at least one parent with bipolar disorder were placed in the bipolar disorder family history group, because a bipolar disorder diagnosis is considered definite. This group included a subset of offspring who also had a parent with a diagnosis of major depressive disorder, which are referred to here as dual-risk offspring (see the section Results).

\section{Actigraphy and Sleep Diary}

Participants wore Micro Motionlogger actigraphs (Ambulatory Monitoring Inc., Ardsley NY) on their nondominant wrist every day for a 2-week period, beginning on a Sunday. They were instructed to remove the actigraph during any water-related activities and while participating in sports. Communication check-ins were made approximately once per week to ensure that there were no difficulties with the protocol or with the actigraphs. Each night was analyzed independently. Additionally, weekdays and weeknights were analyzed separately. Weeknights were defined as Sunday 
through Thursday nights, and weekends were defined as Friday and Saturday nights.

Data were sampled in 60-s epochs, collected in ZeroCrossing Mode, and analyzed with Action W2.7 using the Cole-Kripke scoring algorithm (30). The Cole-Kripke algorithm has been validated in adult and child populations and has been found to be more accurate in estimating TST than the alternative Sadeh algorithm $(24,31)$. From the actigraphy data, we extracted information about the sleep period, TST, SOL, and WASO separately to determine the impact of familial history on each characteristic of sleep. Sleep period was defined as the duration between sleep onset (time fell asleep) and sleep end (morning wake time). TST was the number of actual sleep minutes during the sleep period (i.e., excluding intervening wake episodes).

In accordance with best practice parameters for actigraphy (32, 33), sleep diaries were used to clarify any ambiguous actigraphy data and provided a timeline for when and how long the participants wore the actigraphs. Participants filled out a sleep diary every day during the 2 -week period as recommended by Buysse et al. (21). Participants older than 15 completed a selfreport sleep diary, and parents completed a parent report sleep diary for participants younger than 15. Information collected in the sleep diaries included nightly bedtimes, time required to fall asleep, morning wake times, and daytime naps.

\section{Self-Reported Measures of Sleep Children's Sleep Habits Questionnaire}

This 35-item parent report measure assesses sleep problems in children aged 4-14 years (34). This questionnaire contains eight subscale scores including bedtime resistance, sleep onset delay, sleep duration, sleep anxiety, night waking, parasomnias, sleep disordered breathing, and daytime sleepiness. The Children's Sleep Habits Questionnaire (CSHQ) has been validated in clinical and community populations and has been shown to have good internal consistency and test-retest reliability. Scoring of some items was reversed in order to make a higher score consistently indicative of more disturbed sleep (34).

\section{School Sleep Habits Survey}

This self-report measures sleep/wake habits, sleep patterns during school nights compared to weekends, and daytime functioning of adolescents 15-17 years old (35). The School Sleep Habits Survey (SSHS) consists of 63 questions and has been used in other studies looking at high-risk offspring (7). The SSHS contains three subscales: 1) a depressed mood scale, 2) a sleepiness scale, and 3) a sleep/wake problem behavior scale. The depressed mood scale (36) comprises six items assessing participants' negative emotions in the 2 weeks prior to completing the questionnaire. This scale has high internal reliability and high test-retest reliability for adolescents (35). The sleepiness scale queries the frequency that participants struggled to stay awake in 10 different situations during the 2 weeks prior to completing the questionnaire. The sleep/wake problem scale comprises 10 items examining the frequency of erratic sleep/wake behaviors in the 2 weeks prior to completing the questionnaire (35).

\section{Puberty Measurements}

During adolescence, there are noticeable changes in sleep-wake behaviors due to a variety of psychosocial and biological factors $(37,38)$, which are strongly influenced by the stages of puberty (39). To accurately compare sleep parameters between family history groups, we controlled for puberty onset in our analyses. Pubertal onset was determined by the "Growing and Changing Questionnaire" (GCQ) administered annually in the FORBOW cohort at age 8 and older. The GCQ was based on two measures of pubertal status: Pearson's Puberty Development Scale (PDS) (40) and the Sexual Maturation Scale (SMS) (41, 42). The PDS components of the GCQ asked about body hair, skin changes, growth of facial hair for males, and onset/age of menstruation for females. The SMS section comprises five drawings displaying progressive stages of pubertal development of secondary sexual characteristics ranging from pre-pubertal to post-pubertal. Both the PDS and SMS sections were scored on a 1-5 scale according to Carskadon and Acebo (40) and then averaged to create a composite puberty score. A dichotomous puberty onset score was created to indicate whether each participant was pre- or post-pubertal at the time of sleep assessment. Composite puberty scores $\leq 2$ were scored as pre-pubertal and scores $\geq 3$ were scored as post-pubertal.

\section{Statistical Analysis}

The actigraphy measures of sleep parameters (sleep period, TST, SOL, and WASO) were calculated using Action W2.7 software. Sleep parameters were analyzed separately for weeknights and weekend nights as recommended by Galland et al. (25).

All statistical procedures were conducted using STATA software package, version 15.1 (43). We fitted mixed-effects generalized linear models to test the effect of family history (no diagnosis, major depressive disorder, or bipolar disorder) on sleep period, TST, SOL, and WASO. We included age, sex, pubertal onset, and current diagnosis of Attention Deficit Hyperactivity Disorder (ADHD) as fixed covariates in each model. We accounted for the nonindependence of observations from related individuals and from repeated measures from the same individual by including family and individual identifiers as random effects in the models. We conducted sensitivity analyses controlling for stimulant use, cannabis use, and a current diagnosis of anxiety. We also conducted sensitivity analyses removing offspring with current diagnoses of bipolar disorder $(n=1)$ or major depressive disorder $(n=2)$. We assessed the subscales for the CSHQ and the SSHS for internal consistency using Cronbach's $\alpha$-coefficients. We used mixed-effects generalized linear models with the same fixed covariates and random effects described above for comparisons between family history groups.

\section{RESULTS}

\section{Sample Description}

A total of 86 participants completed the study. Actigraphy data from 13 participants were not included in the analysis due to loss of the actigraph before retrieving the data $(n=3)$, long periods of time without wearing the actigraph $(n=2)$, or corrupted 
TABLE 1 | Demographic and clinical information for the sample.

\begin{tabular}{lllll}
\hline & $\begin{array}{c}\text { Control } \\
\text { offspring } \\
(\boldsymbol{n}=\mathbf{2 8})\end{array}$ & $\begin{array}{c}\text { Offspring of } \\
\text { MDD }(\boldsymbol{n}=\mathbf{2 3})\end{array}$ & $\begin{array}{c}\text { Offspring of } \\
\text { BD }(\boldsymbol{n}=\mathbf{2 2})\end{array}$ & $\begin{array}{c}\text { Total } \\
(\boldsymbol{n}=\mathbf{7 3})\end{array}$ \\
\hline Age, mean (SD) & $11.01(2.97)$ & $11.44(3.44)$ & $\begin{array}{l}12.36(3.32) \\
11.99(3.86)^{1}\end{array}$ & $11.55(3.23)$ \\
Female, $n(\%)$ & $8(29 \%)$ & $11(48 \%)$ & $\begin{array}{l}8(36 \%) \\
4(44 \%)^{1}\end{array}$ & $27(37 \%)$ \\
\hline
\end{tabular}

${ }^{1}$ Demographic and clinical information for dual-risk offspring of one parent with bipolar disorder and one parent with major depressive disorder $(n=9)$.

actigraphy data $(n=8)$. Of the remaining 73 participants (actigraphy completion rate 86.2\%), 23 were offspring of a parent with major depressive disorder ( 9 of whom had both parents with major depressive disorder), 22 were offspring of a parent with bipolar disorder (9 of whom had one parent with bipolar disorder and one parent with major depressive disorder), and 28 were offspring of controls (Table 1). There were 45 pre-pubertal and 28 post-pubertal participants. There were 20 offspring with a current diagnosis: 9 with ADHD, 8 with anxiety, 2 with major depressive disorder, and 1 with bipolar disorder.

\section{Questionnaire Scores}

Among the participants who completed all questionnaires (total completion rate, $75.3 \%$ ), offspring of a parent with bipolar disorder scored significantly higher on the sleep/wake problems scale of the SSHS compared to controls ( $\beta=8.79,95 \%$ CI: 0.83 , $16.75 ; p=0.030)$. There were no significant differences among offspring from the three family history groups for the depressed mood subscale or the sleepiness subscale calculated from the SSHS (see Table 2). There were no group differences for the eight subscales of the CSHQ or the total CSHQ score (see Table 2).

\section{Sleep Diary Results}

As recommended by Meltzer et al (24), we calculated the average self- or parent-reported bedtime and waketime for each family history group (see Table 3). We also calculated the average sleep period as the time between bedtime and wake time. There were no significant differences between family history groups in bedtimes, waketimes, or sleep periods on either weeknights or weekends.

\section{Weeknight Actigraphy Results}

In contrast to the sleep diary results, we found that the offspring of a parent with major depressive disorder had a significantly longer sleep period ( $\beta=25 \mathrm{~min}, 95 \% \mathrm{CI}: 4.54,46.43 ; p=0.017)$ and TST than control offspring on weeknights ( $\beta=26 \mathrm{~min}, 95 \% \mathrm{CI}$ : 3.01 , 49.46; $p=0.027$; see Table 3). We looked separately at the results from offspring with only one or with two parents with major depressive disorder. Those with two parents with a diagnosis of major depressive disorder $(n=9)$ showed a pattern similar to that of the entire group with a longer sleep period than controls that did not reach threshold for statistical significance $(\beta=23 \mathrm{~min}$, 95\% CI: $-5.08,51.53 ; p=0.108)$ and TST $(\beta=26 \mathrm{~min}, 95 \%$
TABLE 2 | Mean and standard deviations of parent-reported Children's Sleep Habits Questionnaire (CSHQ) total score and subscales by family history group for offspring aged 4-14. Mean and standard deviations of self-reported School Sleep Habits Survey (SSHS) subscales by family history group for offspring aged 15-17.

\begin{tabular}{|c|c|c|c|}
\hline & $\begin{array}{l}\text { Offspring } \\
\text { of controls }\end{array}$ & $\begin{array}{l}\text { Offspring } \\
\text { of MDD }\end{array}$ & $\begin{array}{l}\text { Offspring } \\
\text { of BD }\end{array}$ \\
\hline \multicolumn{4}{|l|}{ Children's Sleep Habits } \\
\hline \multicolumn{4}{|l|}{ Questionnaire $^{1}$} \\
\hline \multirow[t]{2}{*}{ Participants $(n)$} & 17 & 14 & 9 \\
\hline & Mean (SD) & Mean (SD) & Mean (SD) \\
\hline \multicolumn{4}{|l|}{ Subscale Item² } \\
\hline Bedtime resistance & $8.50(2.89)$ & $6.64(0.84)$ & $7.89(2.15)$ \\
\hline Sleep onset delay & $1.56(0.71)$ & $1.36(0.63)$ & $1.78(0.67)$ \\
\hline Sleep duration & $4.47(1.74)$ & $3.29(0.47)$ & $4.00(1.58)$ \\
\hline Sleep anxiety & $5.50(2.20)$ & $4.79(1.48)$ & $6.00(2.24)$ \\
\hline Night wakings & $3.50(0.92)$ & $3.21(0.58)$ & $3.56(0.76)$ \\
\hline Parasomnias & $7.28(1.23)$ & $6.36(0.63)$ & $7.89(0.92)$ \\
\hline Sleep disordered breathing & $1.12(0.33)$ & $1.43(0.76)$ & $1.11(0.33)$ \\
\hline Daytime sleepiness & $8.50(2.92)$ & $9.93(2.56)$ & 9.78 (3.93) \\
\hline Total score & $40.44(9.79)$ & $37.00(2.63)$ & $42.00(7.38)$ \\
\hline \multicolumn{4}{|l|}{ School Sleep Habits Survey ${ }^{3}$} \\
\hline \multirow[t]{2}{*}{ Participants $(n)$} & 4 & 3 & $5^{4}$ \\
\hline & Mean (SD) & Mean (SD) & Mean (SD) \\
\hline \multicolumn{4}{|l|}{ Subscale item } \\
\hline Depressed mood scale & $6.90(1.80)$ & $7.00(0.00)$ & $11.00(3.74)$ \\
\hline Sleepiness scale & $11.00(2.00)$ & $12.00(3.46)$ & $12.80(2.68)$ \\
\hline $\begin{array}{l}\text { Sleep/wake problems behavior } \\
\text { scale }\end{array}$ & $19.29(3.15)$ & $23.57(5.96)$ & $28.96^{\star}(6.77)$ \\
\hline
\end{tabular}

${ }^{1}$ Parents of 48 offspring between ages 4 and 14 completed the CSHQ (completion rate, $70.6 \%)$.

${ }^{2}$ The names of the items in this table are those used in the CSHQ.

${ }^{3}$ Twelve offspring between ages 15 and 17 completed the SSHS (completion rate, 92.3\%). ${ }^{4}$ Two offspring in the bipolar family history group were dual-risk offspring who also had a parent with major depressive disorder.

${ }^{*} p<0.05$.

CI: $-5.15,56.54 ; p=0.103)$. Offspring of only one parent with major depressive disorder $(n=14)$ showed a significantly longer sleep period ( $\beta=26.95 \mathrm{~min}, 95 \% \mathrm{CI}: 2.52,51.36 ; p=0.031)$ and a similar, but not statistically significant, increase in TST ( $\beta=$ $26.61 \mathrm{~min}, 95 \% \mathrm{CI}:-0.72,53.94 ; p=0.056)$.

Offspring of a parent with bipolar disorder did not differ significantly from control offspring in their sleep period $(\beta=-3 \mathrm{~min}$, 95\% CI: $-23.99,17.95 ; p=0.777$ ) or TST ( $\beta=-0.79 \mathrm{~min}, 95 \% \mathrm{CI}$ : $-23.86,22.28 ; p=0.946$ ) on weeknights. Average differences between TST calculated from actigraphy and "predicted" TST on weeknights for each family history group are shown in Figure 1. Predicted TST was calculated using a linear prediction from a model based on age, sex, and pubertal status for controls during weeknights.

\section{Weekend Actigraphy Results}

Offspring of a parent with bipolar disorder showed longer, but not statistically significantly different, sleep periods than controls $(\beta=23 \mathrm{~min}, 95 \% \mathrm{CI}:-1.10,49.04 ; p=0.061)$ and TST ( $\beta=20 \mathrm{~min}, 95 \% \mathrm{CI}:-8.59,48.79 ; p=0.170$; see Table 3$)$. The "dual-risk" subgroup of this group (offspring of one parent with bipolar disorder and one with major depressive disorder) showed a significantly longer sleep period compared to controls 
TABLE 3 | Sleep parameters derived from 14 days of actigraphy data by family history group.

\begin{tabular}{|c|c|c|c|c|c|c|c|c|c|c|}
\hline & \multicolumn{2}{|c|}{$\begin{array}{l}\text { Control offspring } \\
\qquad(n=28)\end{array}$} & \multicolumn{2}{|c|}{$\begin{array}{l}\text { Offspring of MDD } \\
\qquad(n=23)\end{array}$} & \multicolumn{2}{|c|}{$\begin{array}{l}\text { Offspring of BD only } \\
\qquad(n=13)\end{array}$} & \multicolumn{2}{|c|}{$\begin{array}{l}\text { Offspring of BD and MDD } \\
\qquad(n=9)\end{array}$} & \multicolumn{2}{|c|}{$\begin{array}{l}\text { All Offspring of BD } \\
\qquad(n=22)\end{array}$} \\
\hline & Mean & $\mathrm{SD}(\min )$ & Mean & $\mathrm{SD}$ (min) & Mean & $\mathrm{SD}(\min )$ & Mean & $\mathrm{SD}$ (min) & Mean & $\begin{array}{l}\mathrm{SD} \\
(\min )\end{array}$ \\
\hline \multicolumn{11}{|l|}{ Weekdays } \\
\hline $\begin{array}{l}\text { Sleep onset } \\
(\mathrm{HH}: \mathrm{MM})\end{array}$ & 22:06 & 117.61 & $22: 05$ & 108.63 & $22: 17$ & 118.08 & $22: 56$ & 155.55 & $22: 33$ & 82.95 \\
\hline $\begin{array}{l}\text { Sleep end } \\
(\mathrm{HH}: \mathrm{MM})\end{array}$ & $7: 21$ & 60.03 & $7: 33$ & 78.45 & $07: 22$ & 94.02 & 8:05 & 131.04 & $7: 40$ & 69.69 \\
\hline \multicolumn{11}{|l|}{$\begin{array}{l}\text { Variables of } \\
\text { Interest }\end{array}$} \\
\hline $\begin{array}{l}\text { Sleep period } \\
\text { (min) }\end{array}$ & 555.36 & 64.81 & $572.08^{*}$ & 68.00 & 559.73 & 66.14 & 534.41 & 84.50 & 538.14 & 74.96 \\
\hline TST (min) & 488.87 & 64.26 & $510.53^{*}$ & 61.34 & 496.03 & 61.51 & 473.17 & 91.61 & 477.83 & 72.01 \\
\hline $\mathrm{SOL}$ (min) & 11.17 & 12.25 & 8.35 & 6.88 & 9.72 & 11.53 & 10.00 & 11.10 & 8.8 & 14.39 \\
\hline WASO (min) & 46.88 & 34.89 & 42.74 & 27.04 & 44.85 & 31.13 & 37.02 & 25.49 & 40.27 & 29.55 \\
\hline \multicolumn{11}{|l|}{ Weekends } \\
\hline $\begin{array}{l}\text { Sleep onset } \\
(\mathrm{HH}: \mathrm{MM})\end{array}$ & $22: 34$ & 138.68 & $22: 39$ & 116.15 & $22: 44$ & 120.35 & 23:01 & 141.21 & $22: 54$ & 83.95 \\
\hline $\begin{array}{l}\text { Sleep end } \\
(\mathrm{HH}: \mathrm{MM})\end{array}$ & $7: 50$ & 123.26 & $7: 59$ & 97.23 & 8:09 & 158.54 & $8: 54$ & 129.43 & $8: 28$ & 68.52 \\
\hline \multicolumn{11}{|l|}{$\begin{array}{l}\text { Variables of } \\
\text { Interest }\end{array}$} \\
\hline $\begin{array}{l}\text { Sleep period } \\
\text { (min) }\end{array}$ & 557.05 & 80.88 & 561.25 & 90.59 & 557.85 & 80.61 & $594.03^{\star}$ & 66.19 & 577.89* & 67.19 \\
\hline TST (min) & 484.18 & 83.58 & 492.73 & 93.63 & 484.65 & 83.31 & 519.43 & 51.56 & 502.46 & 64.95 \\
\hline $\mathrm{SOL}$ (min) & 9.86 & 10.7 & 7.67 & 6.77 & 9.67 & 11.04 & 10.57 & 8.45 & 11.67 & 15.24 \\
\hline WASO (min) & 49.42 & 35.58 & 44.88 & 25.77 & 47.86 & 31.59 & 45.20 & 29.30 & 47.24 & 33.13 \\
\hline
\end{tabular}

${ }^{*} p<0.05$.

on weekend nights ( $\beta=35 \mathrm{~min}, 95 \% \mathrm{CI}$ : $2.55,68.71 ; p=0.035$ ). Offspring of a parent with major depressive disorder did not differ significantly from controls in their sleep period $(\beta=5 \mathrm{~min}$, 95\% CI: $-18.94,28.95 ; p=0.682$ ) or TST ( $\beta=8 \mathrm{~min}, 95 \% \mathrm{CI}$ : $-19.93,35.74 ; p=0.578)$ on weekends.

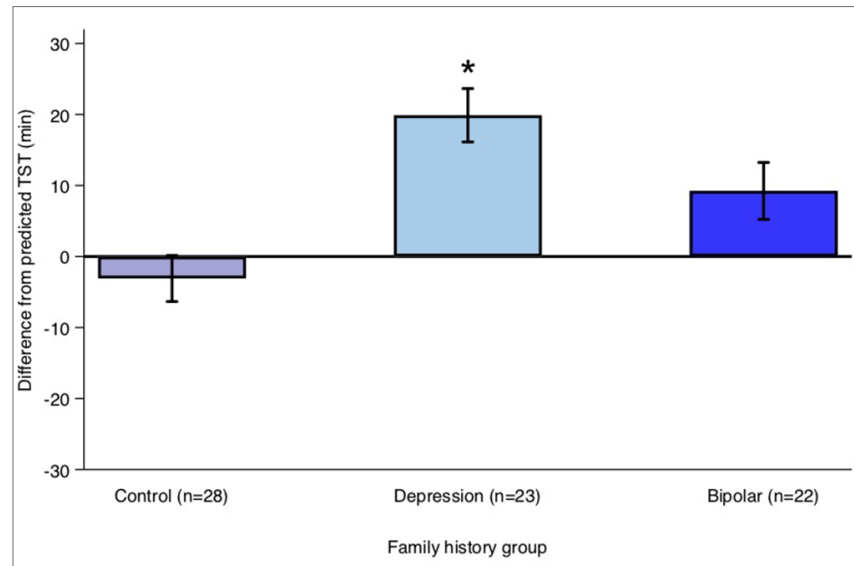

FIGURE 1 | Average difference between actual total sleep time (TST) and predicted TST on weeknights for each family history group. Predicted TST was calculated using a linear prediction from a model predicting TST using age, sex, and pubertal onset for controls during weeknights. Error bars represent the standard error of the mean. Note: ${ }^{*} p<0.05$.

\section{Family History by Weekend Interaction Actigraphy Results}

Offspring of a parent with bipolar disorder showed a significant family history by weekend interaction for TST ( $\beta=27 \mathrm{~min}$, 95\% CI: 7.16, 47.49; $p=0.008$ ) compared to controls (see Figure 2). When this family history group was separated into its two subgroups, dual-risk offspring ( $n=9$; offspring of one parent with bipolar disorder and one with major depressive disorder) showed a family history by weekend interaction for TST ( $\beta=49 \mathrm{~min}, 95 \%$ CI: 22.33, 75.21; $p<0.001$ ), but offspring with one parent with bipolar disorder and a healthy co-parent $(n=13)$ did not ( $\beta=11 \mathrm{~min}, 95 \% \mathrm{CI}$ : -12.12 , 35.31; $p=0.338)$.

The family history by weekend interaction was also significant for sleep period in offspring of a parent with bipolar disorder ( $\beta=36$ min, 95\% CI: 15.76, 57.07; $p=0.001$ ). Dualrisk offspring showed a family history by weekend interaction for sleep period ( $\beta=56 \mathrm{~min}, 95 \%$ CI: $28.73,82.69 ; p<0.001$ ) compared to controls, but offspring with one parent with bipolar disorder and a healthy co-parent showed only a weaker tendency toward the same effect ( $\beta=22 \mathrm{~min}, 95 \% \mathrm{CI}:-1.96$, $46.49 ; p=0.072$ ). Offspring of a parent with major depressive disorder did not show a family history by weekend interaction for either TST ( $\beta=-13 \mathrm{~min}, 95 \%$ CI: $-32.80,6.45 ; p=0.188$ ) or sleep period ( $\beta=-11 \mathrm{~min}, 95 \% \mathrm{CI}:-31.83,8.38 ; p=0.253$ ) compared to control offspring. 


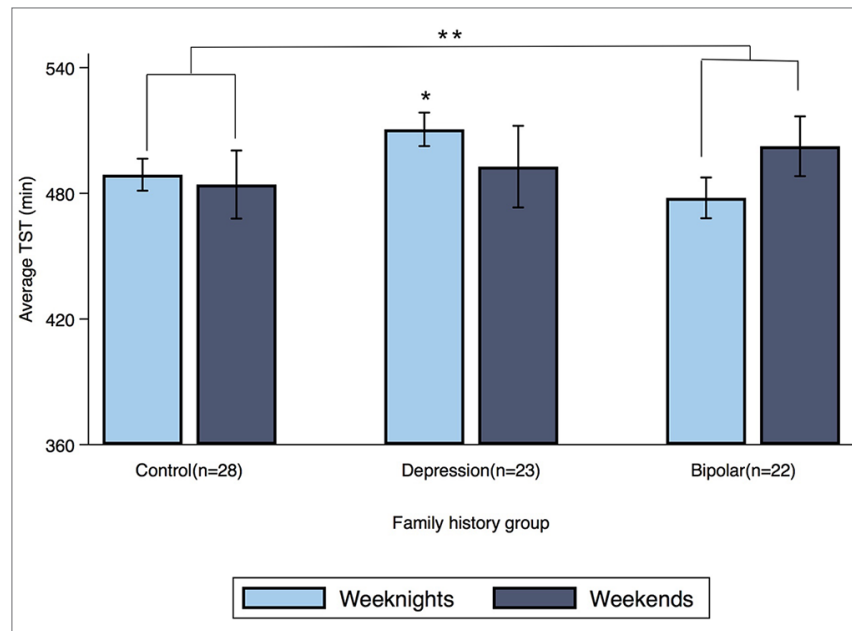

FIGURE 2 | Average TST for weeknights and weekends by family history group. Error bars represent the standard error of the mean. Note: ${ }^{*} p<0.05$, ${ }^{\star \star} p<0.01$.

\section{Sensitivity Analyses}

The significant effects remained unchanged in sensitivity analyses that excluded participants who used stimulant medication or cannabis as determined by self-report measures in the FORBOW study. The significant effects also remained when removing offspring with a current diagnosis of bipolar disorder, major depressive disorder, or anxiety. When offspring with anxiety were removed from the sample, offspring of a parent with bipolar disorder had a significantly longer sleep period on weekends ( $\beta=37 \mathrm{~min}, 95 \%$ CI: 11.71, 62.70; $p=$ $0.004)$. There were no other significant differences in actigraphy results between the groups.

\section{DISCUSSION}

Sleep disturbances, including hypersomnia, insomnia, and irregular sleep patterns, are associated with increased risk of mood disorders $(7,44,45)$, as are sleep features such as higher Rapid Eye Movement sleep density in high-risk individuals (46). Using actigraphy, we found differences in sleep patterns and durations between offspring of parents with mood disorders and control offspring. We found longer sleep periods and TST on weeknights among offspring of a parent with major depressive disorder and weeknight-weekend differences from controls in sleep duration among offspring of a parent with bipolar disorder and a parent with major depressive disorder. It is noteworthy that these effects seen during 2 weeks of actigraphic recording were not matched by any significant group differences in sleep diaries, nor according to the CSHQ. However, we did find significantly more sleep/wake problems in offspring of a parent with bipolar disorder compared to controls as reported on the SSHS.

The increased sleep duration on weekends compared to weeknights among offspring of parents with bipolar disorder is consistent with earlier results based on interviews, which found that at-risk offspring had high rates of sleep disorders (14). Among those at risk for bipolar disorder, a small pilot study suggested that an intervention aimed at stabilizing sleepwake patterns over the week may be beneficial in reducing sleep disturbances (47).

When we analyzed data separately for the dual-risk offspring (one parent with bipolar disorder and one with major depressive disorder) and the offspring of only one parent with bipolar disorder, we found that the dual-risk offspring were driving these weeknight-weekend differences in TST. It is unclear whether this pattern is specific to this diagnostic configuration or reflects additional familial loading of risk resulting from having two parents with any mood disorder diagnosis. However, our results comparing offspring with one or two parents with major depressive disorder do not support a generalized risk of having both parents with any mood disorder diagnosis. Rather, they highlight a potential specific risk associated with having one parent with bipolar disorder and one parent with major depressive disorder. In this context, the fact that major depressive disorder is sometimes re-diagnosed as bipolar disorder allows for the possibility that some of these families will include two parents with bipolar disorder. Future follow-ups may permit assessment of this possibility. This result suggests that while altered weeknight-weekend sleep patterns may be an early indicator of increased risk for development of bipolar disorder, those who have two parents with this specific diagnostic configuration may be at greater risk.

Offspring of parents with major depression had longer sleep periods and TST during weeknights than controls. Most treatments for sleep disturbances associated with major depressive disorder to date have focused on treating insomnia (2). Our results suggest that hypersomnia may be an early indicator of increased risk for depression in some children and could be a relevant target for future sleep-based interventions for individuals at high familial risk.

\section{Strengths and Limitations}

Our study benefited from the inclusion of offspring at high familial risk for multiple forms of mood disorders across a broad age range and the use of an objective measure of sleep quality in a relatively large sample of high-risk youth. Actigraphic recording for 2 weeks allowed us to assess weeknight-weekend differences, which proved to be informative. Questionnaire data, which are known to detect sleep abnormalities in clinical populations, did not reproduce the results found with actigraphy in this high-risk population, with the exception of identifying increased sleep problems in offspring of a parent with bipolar disorder. These findings suggest caution in interpreting results of questionnaires and short-term actigraphy data in at-risk children.

Several factors in this study limit the strength of our conclusions. Because of the number of participants in each group, we could not compare results among groups at different ages across the study sample. We did, however, control for both age and pubertal status in our statistical analyses. Because of power limits, we also reported 
our results as nominal significance levels. It should be noted that there were only nine participants in the dual-risk group; thus, the results for this subgroup should be considered preliminary. Overall, our results suggest that familial risk has an effect on offspring's sleep patterns; however, it is not possible to distinguish whether parentally contributed genes, the impact of parental illness on the family/offspring, or an interaction of the two contributes to these differences, especially in dual-risk offspring.

The FORBOW study is designed as a cohort with ongoing recruitment of children and adolescents. We anticipate adding additional participants to each of the groups, with a special focus on increasing the number of dual-risk offspring. The cohort will be followed for at least a 5-year period, which will allow for determination of the clinical outcomes for these children and adolescents at high risk and for the evaluation of the predictive power of early sleep-wake characteristics in the development of mood disorders. The results should also facilitate development of early sleep interventions tailored to the characteristics of different at-risk groups and the evaluation of their effects on risk of illness.

\section{ETHICS STATEMENT}

The protocol was approved by the Research Ethics Board of the Nova Scotia Health Authority, in conformity with the Canadian Tri-Council Policy Statement 2: Ethical Conduct of Research involving Humans (2014). We obtained informed consent from participants who had the capacity to provide it. For participants who did not have the capacity to make an informed decision, a parent or guardian provided written informed consent and the participant provided assent.

\section{AUTHOR CONTRIBUTIONS}

All authors listed have contributed sufficiently to the project to be included as authors, and all those who are qualified to be authors are listed in the author byline. DW contributed substantially to the conception and design, acquisition

\section{REFERENCES}

1. Robillard R, Hermens DF, Naismith SL, White D, Rogers NL, Ip TKC, et al. Ambulatory sleep-wake patterns and variability in young people with emerging mental disorders. J Psychiatry Neurosci (2015) 40(1):28-37. doi: 10.1503/jpn.130247

2. Franzen PL, Buysse DJ. Sleep disturbances and depression: risk relationships for subsequent depression and therapeutic implications. Dialogues Clin Neurosci (2008) 10(4):473-81.

3. Mindell JA, Owens JA, Carskadon MA. Developmental features of sleep. Child Adolesc Psychiatr Clin N Am (1999) 8(4):695-725. doi: 10.1016/ S1056-4993(18)30149-4

4. Lovato N, Gradisar M. A meta-analysis and model of the relationship between sleep and depression in adolescents: recommendations for future research and clinical practice. Sleep Med Rev (2014) 18(6):521-9. doi: 10.1016/j.smrv.2014.03.006

5. Vanderlind WM, Beevers CG, Sherman SM, Trujillo LT, McGeary JE, Matthews MD, et al. Sleep and sadness: exploring the relation among of data, and analysis and interpretation of data. DW also drafted the article and gave final approval of the version to be published. JM contributed to the conception and design and to the acquisition of data, revised the manuscript critically for important intellectual content, and gave final approval of the version to be published. AZ contributed to the analysis and interpretation of data, revised the manuscript critically for important intellectual content, and gave final approval of the version to be published. JC contributed to the acquisition of data and gave final approval of the version to be published. RU contributed substantially to the conception and design, acquisition of data, and analysis and interpretation of data; revised the manuscript critically for important intellectual content; and gave final approval of the version to be published. BR contributed substantially to the conception and design and to the analysis and interpretation of data, revised the manuscript critically for important intellectual content, and gave final approval of the version to be published.

\section{FUNDING}

This project was supported by funding from the Dalhousie Psychiatry Research Fund, Lindsay Family Graduate Scholarship, Canada Research Chairs Program (award number 231397), the Canadian Institutes of Health Research (grant reference numbers 124976, 142738, and 148394), the Brain \& Behavior Research Foundation (NARSAD) Independent Investigator Grant 24684, Nova Scotia Health Research Foundation (grants 275319, 1716, and 353892), the Dalhousie Medical Research Foundation, and the Natural Sciences and Engineering Research Council of Canada (grant RGPIN-305).

\section{ACKNOWLEDGEMENTS}

We would like to thank members of the Corkum LABS for their assistance in developing the sleep diary and Sarah Brine for her help with the actigraphy analysis.

sleep, cognitive control, and depressive symptoms in young adults Sleep Med (2014) 15(1):144-9. doi: 10.1016/j.sleep.2013.10.006

6. Lunsford-Avery JR, Judd CM, Axelson DA, Miklowitz DJ. Sleep impairment, mood symptoms, and psychosocial functioning in adolescent bipolar disorder. Psychiatry Res (2012) 200(2-3):265-71. doi: 10.1016/j. psychres.2012.07.037

7. Levenson JC, Axelson DA, Merranko J, Angulo M, Goldstein TR, Goldstein BI, et al. Differences in sleep disturbances among offspring of parents with and without bipolar disorder: association with conversion to bipolar disorder. Bipolar Disord (2015) 17(8):836-48. doi: 10.1111/bdi.12345

8. Lee YJ, Cho S-J, Cho IH, Jang JH, Kim SJ. The relationship between psychotic-like experiences and sleep disturbances in adolescents. Sleep Med (2012) 13(8):1021-7. doi: 10.1016/j.sleep.2012.06.002

9. Gregory AM, Sadeh A. Sleep, emotional and behavioral difficulties in children and adolescents. Sleep Med Rev (2012) 16(2):129-36. doi: 10.1016/j. smrv.2011.03.007

10. Armstrong JM, Ruttle PL, Klein MH, Essex MJ, Benca RM. Associations of child insomnia, sleep movement, and their persistence with mental health 
symptoms in childhood and adolescence. Sleep (2014) 37(5):901-9. doi: 10.5665/sleep.3656

11. Duffy A, Alda M, Hajek T, Sherry SB, Grof P. Early stages in the development of bipolar disorder. J Affect Disord (2010) 121(1-2):127-35. doi: 10.1016/j. jad.2009.05.022

12. Rasic D, Hajek T, Alda M, Uher R. Risk of mental illness in offspring of parents with schizophrenia, bipolar disorder, and major depressive disorder: a meta-analysis of family high-risk studies. Schizophr Bull (2014) 40(1):28. doi: $10.1093 /$ schbul/sbt114

13. Uher R. Gene-environment interactions in common mental disorders: an update and strategy for a genome-wide search. Soc Psychiatry Psychiatr Epidemiol (2014) 49(1):3-14. doi: 10.1007/s00127-013-0801-0

14. Duffy A. The early natural history of bipolar disorder: what we have learned from longitudinal high-risk research. Can J Psychiatry (2010) 55(8):477-85. doi: $10.1177 / 070674371005500802$

15. de Kloet ER, Joëls M, Holsboer F. Stress and the brain: from adaptation to disease. Nat Rev Neurosci (2005) 6(6):463-75. doi: 10.1038/nrn1683

16. McGorry PD, Killackey E, Yung A. Early intervention in psychosis: concepts, evidence and future directions. World Psychiatry (2008) 7(3):148-56. doi: 10.1002/j.2051-5545.2008.tb00182.x

17. Taylor AK, Netsi E, O'Mahen H, Stein A, Evans J, Pearson RM. The association between maternal postnatal depressive symptoms and offspring sleep problems in adolescence. Psychol Med (2017) 47(3):451-9. doi: $10.1017 /$ S0033291716002427

18. Pearson RM, Evans J, Kounali D, Lewis G, Heron J, Ramchandani PG, et al. Maternal depression during pregnancy and the postnatal period: risks and possible mechanisms for offspring depression at 18 years. JAMA Psychiatry (2013) 70(12):1312. doi: 10.1001/jamapsychiatry.2013.2163

19. Milhiet V, Boudebesse C, Bellivier F, Drouot X, Henry C, Leboyer M, et al. Circadian abnormalities as markers of susceptibility in bipolar disorders. Front Biosci (Schol Ed) (2014) 6:120-37. doi: 10.2741/s419

20. Sebela A, Novak T, Kemlink D, Goetz M. Sleep characteristics in child and adolescent offspring of parents with bipolar disorder: a case control study. BMC Psychiatry (2017) 17:199. doi: 10.1186/s12888-017-1361-8.

21. Buysse DJ, Ancoli-Israel S, Edinger JD, Lichstein KL, Morin CM. Recommendations for a standard research assessment of insomnia. Sleep (2006) 29(9):1155-73. doi: 10.1093/sleep/29.9.1155

22. Sadeh A, Hauri PJ, Kripke DF, Lavie P. The role of actigraphy in the evaluation of sleep disorders. Sleep (1995) 18(4):288-302. doi: 10.1093/sleep/18.4.288

23. Martin JL, Hakim AD. Wrist actigraphy. Chest (2011) 139(6):1514-27. doi: 10.1378/chest.10-1872

24. Meltzer LJ, Walsh CM, Traylor J, Westin AML. Direct comparison of two new actigraphs and polysomnography in children and adolescents. Sleep (2012) 35(1):159-66. doi: 10.5665/sleep.1608

25. Galland BC, Short MA, Terrill P, Rigney G, Haszard JJ, Coussens S, et al. Establishing normal values for pediatric nighttime sleep measured by actigraphy: a systematic review and meta-analysis. Sleep (2018) 41(4):1-16. doi: 10.1093/sleep/zsy017

26. Hysing M, Sivertsen B, Stormark KM, O'Connor RC. Sleep problems and self-harm in adolescence. Br J Psychiatry (2015) 207(4):306-12. doi: 10.1192/ bjp.bp.114.146514

27. Inderkum AP, Tarokh L. High heritability of adolescent sleep-wake behavior on free, but not school days: a long-term twin study. Sleep (2018) 41(3):1-9. doi: 10.1093/sleep/zsy004

28. Uher R, Cumby J, MacKenzie LE, Morash-Conway J, Glover JM, Aylott A, et al. A familial risk enriched cohort as a platform for testing early interventions to prevent severe mental illness. BMC Psychiatry (2014) 14:344. doi: 10.1186/s12888-014-0344-2

29. First MB, Williams JBW, Karg RS, Spitzer RL. Structured Clinical Interview for DSM-5-Research Version (SCID-5 for DSM-5, Research Version; SCID-5-RV). Arlington, VA, American Psychiatric Association (2015).

30. Cole RJ, Kripke DF, Gruen W, Mullaney DJ, Gillin JC. Automatic sleep/wake identification from wrist activity. Sleep (1992) 15(5):461-9. doi: 10.1093/ sleep/15.5.461
31. Quante M, Kaplan ER, Cailler M, Rueschman M, Wang R, Weng J, et al. Actigraphy-based sleep estimation in adolescents and adults: a comparison with polysomnography using two scoring algorithms. Nat Sci Sleep (2018) 10:13-20. doi: 10.2147/NSS.S151085

32. Ancoli-Israel S, Cole R, Alessi C, Chambers M, Moorcroft W, Pollak CP. The role of actigraphy in the study of sleep and circadian rhythms. Sleep (2003) 26(3):342-92. doi: 10.1093/sleep/26.3.342

33. Acebo C, LeBourgeois MK. Actigraphy. Respir Care Clin N Am (2006) 12(1):23-30. doi: 10.1016/j.rcc.2005.11.010 viii.

34. Owens JA, Spirito A, McGuinn M. The Children's Sleep Habits Questionnaire (CSHQ): psychometric properties of a survey instrument for school-aged children. Sleep (2000) 23(8):1-9. doi: 10.1093/sleep/ 23.8.1d

35. Wolfson AR, Carskadon MA. Sleep schedules and daytime functioning in adolescents. Child Dev (1998) 69(4):875-87. doi: 10.1111/j.1467-8624.1998. tb06149.x

36. Kandel DB, Davies M. Epidemiology of depressive mood in adolescents: an empirical study. Arch Gen Psychiatry (1982) 39(10):1205-12. doi: 10.1001/ archpsyc.1982.04290100065011

37. Carskadon MA, Vieira C, Acebo C. Association between puberty and delayed phase preference. Sleep (1993) 16(3):258-62. doi: 10.1093/sleep/16.3.258

38. Wolfson AR, Carskadon MA. Understanding adolescent's sleep patterns and school performance: a critical appraisal. Sleep Med Rev (2003) 7(6):491-506. doi: 10.1016/S1087-0792(03)90003-7

39. Laberge L, Petit D, Simard C, Vitaro F, Tremblay RE, Montplaisir J. Development of sleep patterns in early adolescence. J Sleep Res (2001) 10(1):59-67. doi: 10.1046/j.1365-2869.2001.00242.x

40. Carskadon MA, Acebo C. A self-administered rating scale for pubertal development. J Adolesc Health (1993) 14(3):190-5. doi: 10.1016/1054-139X(93)90004-9

41. Marshall WA, Tanner JM. Variations in pattern of pubertal changes in girls. Arch Dis Child (1969) 44(235):291-303. doi: 10.1136/adc.44.235.291

42. Marshall WA, Tanner JM. Variations in the pattern of pubertal changes in boys. Arch Dis Child (1970) 45(239):13-23. doi: 10.1136/adc.45.239.13

43. StataCorp. Stata Statistical Software: Release 15. College Station, TX: StataCorp LLC (2017).

44. Breslau N, Roth T, Rosenthal L, Andreski P. Sleep disturbance and psychiatric disorders: a longitudinal epidemiological study of young adults. Biol Psychiatry (1996) 39(6):411-8. doi: 10.1016/ 0006-3223(95)00188-3

45. Kaplan KA, Harvey AG. Hypersomnia across mood disorders: a review and synthesis. Sleep Med Rev (2009) 13(4):275-85. doi: 10.1016/j. smrv.2008.09.001

46. Modell S, Ising M, Holsboer F, Lauer CJ. The Munich vulnerability study on affective disorders: premorbid polysomnographic profile of affected high-risk probands. Biol Psychiatry (2005) 58(9):694-9. doi: 10.1016/j. biopsych.2005.05.004

47. Goldstein TR, Fersch-Podrat R, Axelson DA, Gilbert A, Hlastala SA, Birmaher B, et al. Early intervention for adolescents at high risk for the development of bipolar disorder: pilot study of Interpersonal and Social Rhythm Therapy (IPSRT). Psychotherapy (2014) 51(1):180-9. doi: 10.1037/ a0034396

Conflict of Interest Statement: The authors declare that the research was conducted in the absence of any commercial or financial relationships that could be construed as a potential conflict of interest.

Copyright (c) 2019 Wescott, Morash-Conway, Zwicker, Cumby, Uher and Rusak. This is an open-access article distributed under the terms of the Creative Commons Attribution License (CC BY). The use, distribution or reproduction in other forums is permitted, provided the original author $(s)$ and the copyright owner(s) are credited and that the original publication in this journal is cited, in accordance with accepted academic practice. No use, distribution or reproduction is permitted which does not comply with these terms. 